DIW BERLIN

Discussion

Papers
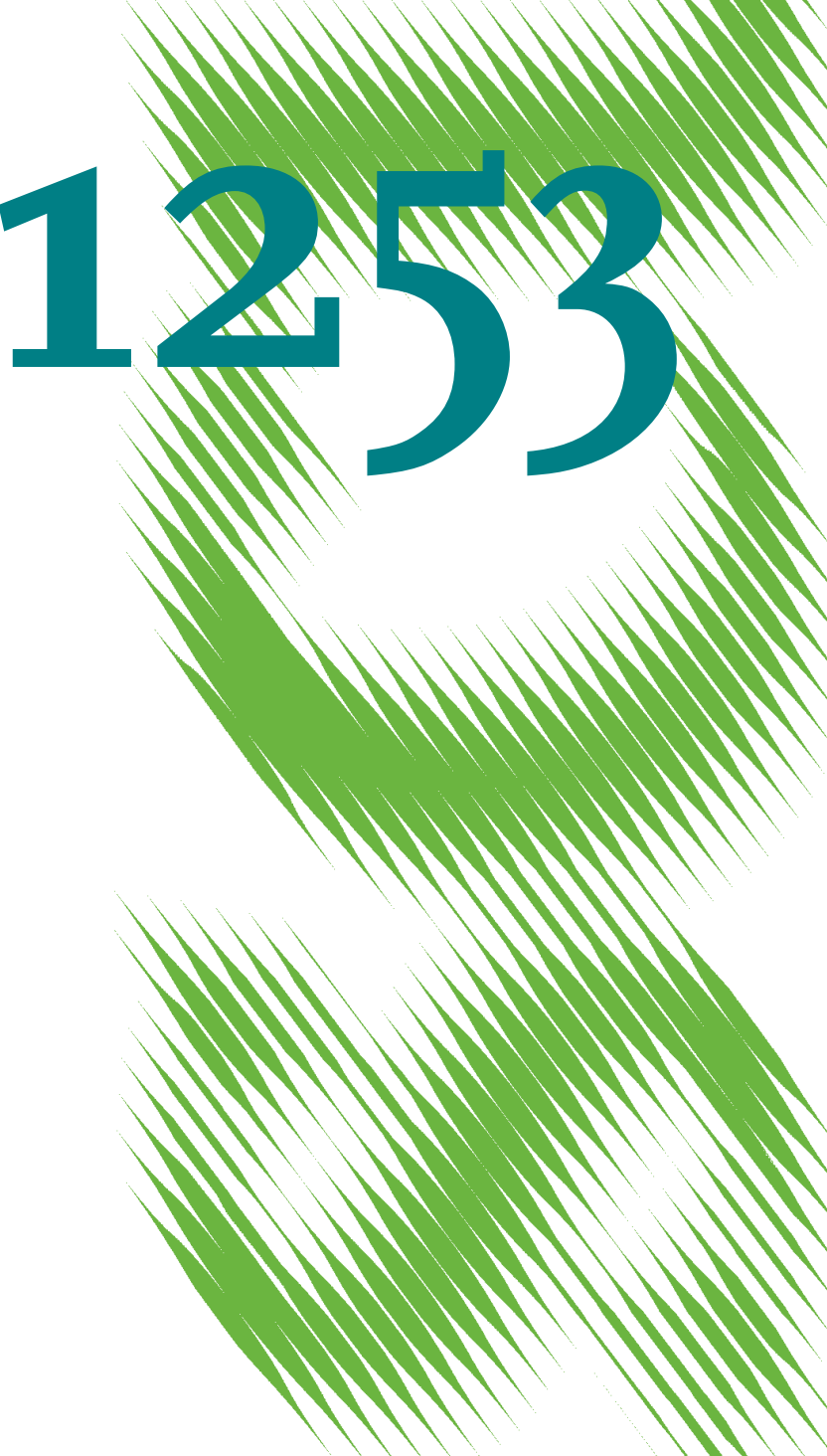

Endogenous Investment Decisions in Natural Gas Equilibrium Models with Logarithmic Cost Functions 
Opinions expressed in this paper are those of the author(s) and do not necessarily reflect views of the institute.

IMPRESSUM

(C) DIW Berlin, 2012

DIW Berlin

German Institute for Economic Research

Mohrenstr. 58

10117 Berlin

Tel. $+49(30) 89789-0$

Fax +49 (30) $89789-200$

http://www.diw.de

ISSN print edition $1433-0210$

ISSN electronic edition 1619-4535

Papers can be downloaded free of charge from the DIW Berlin website:

http://www.diw.de/discussionpapers

Discussion Papers of DIW Berlin are indexed in RePEc and SSRN:

http://ideas.repec.org/s/diw/diwwpp.html

http://www.ssrn.com/link/DIW-Berlin-German-Inst-Econ-Res.html 


\title{
Endogenous investment decisions in natural gas equilibrium models with logarithmic cost functions*
}

\author{
Daniel Huppmann \\ German Institute for Economic Research (DIW Berlin) \\ Mohrenstraße 58, 10117 Berlin, Germany \\ dhuppmann@diw.de \\ Corrected version \\ November 29, 2012
}

\begin{abstract}
The liberalisation of the natural gas markets and the importance of natural gas as a transition fuel to a low-carbon economy have led to the development of several large-scale equilibrium models in the last decade. These models combine long-term market equilibria and investments in infrastructure while accounting for market power by certain suppliers. They are widely used to simulate market outcomes given different scenarios of demand and supply development, environmental regulations and investment options.

In order to capture the specific characteristics of natural gas production, most of these models apply a logarithmic production cost function. However, no model has so far combined this cost function type with endogenous investment decisions in production capacity. Given the importance of capacity constraints in the determination of the natural gas supply, this is a serious shortcoming of the current literature. This paper provides a proof that combining endogenous investment decisions and a logarithmic cost function yields indeed a convex minimization problem, paving the way for an important extension of current state-of-the-art equilibrium models.
\end{abstract}

Keywords: natural gas, equilibrium model, endogenous investment, capacity expansion, logarithmic cost function

JEL Codes: C61, Q41, L71

\footnotetext{
${ }^{*}$ I would like to thank Franziska Holz and Philipp M. Richter for their helpful comments and suggestions for improvements of this work as well as Ibrahim Abada for pointing out two typographical errors in an earlier version.
} 


\section{Introduction}

The global natural gas market has changed significantly over the last two decades. Liberalisation of natural gas markets in Europe has in part led to the gradual replacement of long-term contracts by short-term spot markets. Stringent carbon dioxide $\left(\mathrm{CO}_{2}\right)$ emission constraints are intended to induce a shift from coal and oil to comparatively clean natural gas (EC, 2011). Unconventional reserves are a game-changer in North America and maybe other regions, with the OECD speaking of a "golden age of gas" (IEA, 2011). Last but not least, the question of supply security and European dependence on a small number of suppliers for a substantial share of its imports arises frequently (cf. Leveque et al. 2010).

These factors have led to a considerable interest in modelling the future development of natural gas markets. A number of equilibrium models have been developed to provide numerical analysis of different scenarios regarding supply and demand patterns, environmental regulation and infrastructure investment options. These efforts were made possible by theoretical and algorithmic advances in solving such problems (Mathiesen, 1985, Harker and Pang, 1990, Ferris and Munson, 2000: Facchinei and Pang, 2003). One early example of applied work can be found in Mathiesen et al. (1987).

Two large-scale natural gas equilibrium models developed in the past decade stand out in particular: the GASTALE model, developed by ECN (Lise and Hobbs, 2008), and the World Gas Model (WGM), joint work by the University of Maryland and DIW Berlin (Egging et al., 2010) 11 These models share a number of characteristics: they are spatial partial equilibrium models with a detailed geographic disaggregation, allowing for analysis and comparison of different pipeline and LNG export/import options; they consider seasonality within a year and explicitly model storage to shift natural gas between low- and high-demand seasons; they are multi-year models and endogenously determine optimal investment in infrastructure; and they allow for oligopolistic behaviour by (a subset of) suppliers, i.e., Cournot competition. Both models also apply a logarithmic cost function, as first proposed by Golombek et al. (1995), in order to capture the specific characteristics of natural gas production: sharply increasing costs when producing close to full capacity.

However, neither of the models allows for endogenous investment in production capacity; instead, the production capacity in future periods is defined exogenously. Given that production capacity is a significant determinant of results and that these models simulate price and quantity trajectories for several decades into the future, this omission is certainly a major drawback. It is owed, in all likelihood, to the rather complicated functional

\footnotetext{
${ }^{1}$ Both models were published in different versions and used extensively for scenario simulations; only one recent publication for each model is cited here.
} 
form when including investment decision variables in the logarithmic cost function. This paper provides the proof that this extension yields a convex problem, which is a prerequisite for solving this problem as an equilibrium model.

Let me also mention two more recent natural gas models: the GaMMES model was developed by EDF and IFPEN (Abada et al., 2012). In contrast to the models presented above, it distinguishes between spot market sales and long-term contracts. It also assumes a slightly different formulation of the logarithmic cost function: production costs are not increasing relative to capacity utilization, as in the other models, but relative to remaining reserves. This is an interesting approach, but differs from what is discussed in this work. EWI Cologne is currently developing the COLUMBUS model (Hecking and Panke, 2012). It is - at this stage - formulated as a linear complementarity problem and does not use a logarithmic cost function.

All these models are formulated as Mixed Complementarity Problem (MCP). The optimization problems of different players subject to engineering and other constraints are solved simultaneously by deriving their respective Karush-Kuhn-Tucker (KKT) conditions, combined with market clearing constraints. The MCP framework is convenient for this type of exercise, as it allows to include Cournot market power for certain suppliers, in contrast to welfare maximization or cost minimization problems. In addition, these models can easily be extended to include stochasticity (e.g., Gabriel et al., 2009) or two-level problems such as Stackelberg competition (e.g., Siddiqui and Gabriel, 2012).

\section{Mathematical formulation}

Assume a supplier with decision variables $q_{y}$ (production quantity) and $e_{y}$ (production capacity expansion/investment). The periods are denoted by $y \in\{1, \ldots, \bar{y}\}$. In order to keep the notation concise, $y$ denotes both a period as well as its position in the set. Hence, $\bar{y}$ stands for both the last period as well as the number of periods in the set. Following this logic, I use $y^{\prime}<y$ for "all periods $y^{\prime}$ prior to period $y$ " in sums and indices, and $y^{\prime}>y$ for the inverse statement. The price at which the produced quantity is sold is denoted by $p_{y}$, and the initial production capacity is $\bar{q}$.

Production $\operatorname{costs} c_{y}(\cdot)$ are determined by a logarithmic cost function as introduced by Golombek et al. (1995) related to capacity utilization (see Equation (3a below). This function is illustrated in Figure 1. Marginal production costs increase sharply when operating close to capacity. Hence, if capacity is expanded, marginal production costs for the same quantity decrease.

In line with the literature, capacity investment costs are assumed to be linear. The parameters of the cost function are denoted by greek letters 


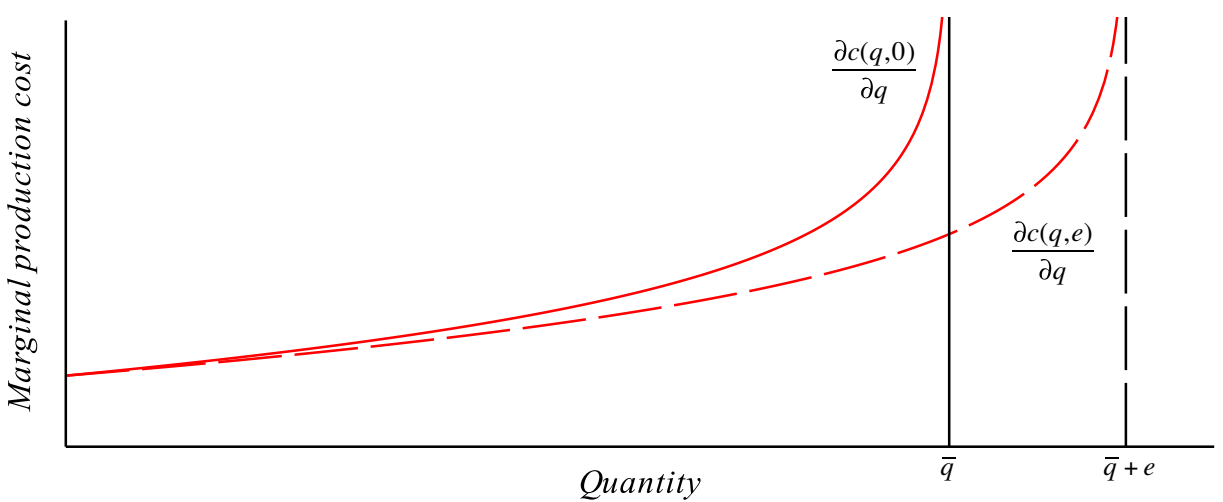

Figure 1: Illustration of the marginal cost function (production capacity $\bar{q}$ ) without investment $(-)$ and with additional investment $e(--)$

and may vary by period: $\alpha_{y}, \beta_{y}, \gamma_{y}$ are the parameters for the production cost function; $\kappa_{y}$ is the (linear) unit production capacity investment cost. All cost parameters are positive. Discounting of future profits may be implicitly included in the price and cost parameters. For now, I abstract from Cournot market power and other considerations such as reserve horizon or maximum investment constraints. These extensions are briefly discussed below.

The profit maximization problem of the supplier can then be written as follows, converted to a minimization problem:

$$
\begin{array}{rl}
\min _{q, e} & f(q, e)=\sum_{y}-p_{y} q_{y}+c_{y}\left(q_{y}, e_{1}, \ldots, e_{y-1}\right)+\kappa_{y} e_{y} \\
\text { s.t. } & q, e \in \mathbb{R}_{+}^{\bar{y}}
\end{array}
$$

This yields the following Karush-Kuhn-Tucker conditions:

$$
\begin{array}{r}
-p_{y}+\frac{\partial c_{y}(\cdot)}{\partial q_{y}} \geq 0 \perp q_{y} \geq 0 \\
\sum_{y^{\prime}>y} \frac{\partial c_{y^{\prime}}(\cdot)}{\partial e_{y}}+\kappa_{y} \geq 0 \perp e_{y} \geq 0
\end{array}
$$

It is straightforward to see that there will never be investment in the last period; the KKT condition reduces to $0+\kappa_{y} \geq 0$, implying $e_{y}=0$ if $\kappa_{y}>0$. This variable and the associated equation can thus be omitted from further consideration.

The production cost function and its partial derivatives are listed below. In order to make the notation more concise, the sum of previous investments, 
$\sum_{y^{\prime}<y} e_{y^{\prime}}$, is replaced by $e(y)$ for the remainder of this work.

$$
\begin{array}{rlr}
c_{y}(\cdot)= & \left(\alpha_{y}+\gamma_{y}\right) q_{y}+\beta q_{y}^{2} \\
& +\gamma_{y}\left(\bar{q}+e(y)-q_{y}\right) \ln \left(1-\frac{q_{y}}{\bar{q}+e(y)}\right) & \\
\frac{\partial c_{y}(\cdot)}{\partial q_{y}}= & \alpha_{y}+2 \beta_{y} q_{y}-\gamma_{y} \ln \left(1-\frac{q_{y}}{\bar{q}+e(y)}\right) & \\
\frac{\partial c_{y}(\cdot)}{\partial e_{\hat{y}}}= & \gamma_{y} \ln \left(1-\frac{q_{y}}{\bar{q}+e(y)}\right)+\gamma_{y} \frac{q_{y}}{\bar{q}+e(y)} & \\
\frac{\partial^{2} c_{y}(\cdot)}{\partial q_{y}^{2}}= & 2 \beta_{y}+\gamma_{y} \frac{1}{\bar{q}+e(y)-q_{y}} & \text { if } \hat{y}<y \\
\frac{\partial^{2} c_{y}(\cdot)}{\partial e_{\hat{y}} \partial e_{\tilde{y}}}= & \gamma_{y} \frac{q_{y}^{2}}{\left(\bar{q}+e(y)-q_{y}\right)(\bar{q}+e(y))^{2}} & \text { if } \hat{y}<y \wedge \tilde{y}<y \\
\frac{\partial^{2} c_{y}(\cdot)}{\partial q_{y} \partial e_{\hat{y}}}= & -\gamma_{y} \frac{q_{y}}{\left(\bar{q}+e(y)-q_{y}\right)(\bar{q}+e(y))} & \text { if } \hat{y}<y
\end{array}
$$

Given this cost function and assuming $\gamma_{y}>0 \forall y$, marginal production costs tend to infinity when the produced quantity tends to initial capacity plus expansions in previous periods. Hence, production quantity $q_{y}$ is implicitly bounded by capacity. Mathematically speaking, for any $p_{y}>0$, there exists a quantity $q_{y}$ with $\frac{\partial c_{y}(\cdot)}{\partial q_{y}} \geq p_{y}$ and $q_{y}<\bar{q}+e(y)$. Hence, an explicit production capacity condition is not required as a constraint in the optimization problem (1).

Marginal production $\operatorname{costs} \frac{\partial c_{y}(\cdot)}{\partial q_{y}}$ are non-negative, while the effect of an expansion on costs $\frac{\partial c_{y}(\cdot)}{\partial e_{y}}$ is non-positive. ${ }^{2}$ A capacity expansion today reduces total cost in subsequent periods periods for any fixed quantity (cf. Figure 11. In contrast, in a model with linear or quadratic production cost functions and endogenous capacity expansion, only the dual (or shadow price) to the capacity constraint in future periods determines the investment; hence, only the possibility of producing a higher quantity drives capacity expansion decisions.

Theorem 1. Any solution to the KKT system (2) is a global optimum of the supplier profit maximization problem (1).

Proof. Sufficiency of the KKT conditions can be established by showing convexity of the objective function $f(q, e)$, which is equivalent to its Hessian matrix being positive semidefinite for any feasible vector $(q, e)$. As shown earlier, investment in the last period can be omitted. The matrix has the

\footnotetext{
${ }^{2}$ Note that capacity utilization $\frac{q_{y}}{\bar{q}+e(y)} \in[0,1]$ for any feasible quantity $q_{y}$. Since $\ln (1-x)+x \leq 0 \forall x \in[0,1]$, that statement holds.
} 
following form:

$$
\begin{aligned}
& H(f(q, e))=\left(\begin{array}{cc}
f_{q q} & f_{q e} \\
\left(f_{q e}\right)^{T} & f_{e e}
\end{array}\right)=
\end{aligned}
$$

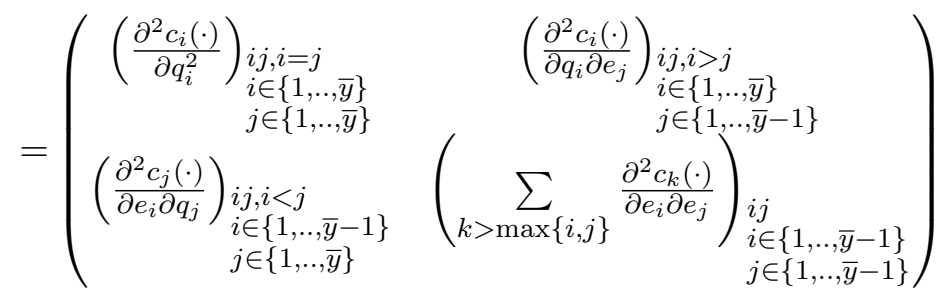

This matrix is symmetric and all its diagonal entries are positive, but it is not necessarily diagonally dominant; hence, we cannot apply a standard result to show positive semidefiniteness. Nevertheless, the partial derivatives of the cost function have a certain structure which can be exploited to show $x^{T} M x \geq 0$ for all $x \in \mathbb{R}^{n}$.

Define $x \in \mathbb{R}^{2 \bar{y}-1}$ as follows: $x=\left[\left(a_{y}\right),\left(b_{y}\right)\right]^{T}$. This is to exploit the different interpretation of the production vs. expansion variables.

$$
\begin{aligned}
& {\left[\begin{array}{l}
\left(a_{y}\right)_{y \in\{1, . ., \bar{y}\}} \\
\left(b_{y}\right)_{y \in\{1, . ., \bar{y}-1\}}
\end{array}\right]^{T} H(f(q, e))\left[\begin{array}{l}
\left(a_{y}\right)_{y \in\{1, \ldots, \bar{y}\}} \\
\left(b_{y}\right)_{y \in\{1, \ldots, \bar{y}-1\}}
\end{array}\right]=} \\
& =\left[\begin{array}{l}
\left(a_{y} \frac{\partial^{2} c_{y}(\cdot)}{\partial q_{y}^{2}}+\sum_{\hat{y}<y} b_{\hat{y}} \frac{\partial^{2} c_{y}(\cdot)}{\partial e_{\hat{y}} \partial q_{y}}\right)_{y \in\{1, \ldots, \bar{y}\}} \\
\left(\sum_{\hat{y}>y} a_{\hat{y}} \frac{\partial^{2} c_{\hat{y}}(\cdot)}{\partial q_{\hat{y}} \partial e_{y}}+\sum_{\hat{y}} b_{\hat{y}} \sum_{k>\max \{y, \hat{y}\}} \frac{\partial^{2} c_{k}(\cdot)}{\partial e_{y} \partial e_{\hat{y}}}\right)_{y \in\{1, \ldots, \bar{y}-1\}}
\end{array}\right]^{T}\left[\begin{array}{l}
\left(a_{y}\right)_{y \in\{1, \ldots, \bar{y}\}} \\
\left(b_{y}\right)_{y \in\{1, \ldots, \bar{y}-1\}}
\end{array}\right]= \\
& =\sum_{y} a_{y}^{2} \frac{\partial^{2} c_{y}(\cdot)}{\partial q_{y}^{2}}+\sum_{y} a_{y} \sum_{\hat{y}<y} b_{\hat{y}} \frac{\partial^{2} c_{y}(\cdot)}{\partial e_{\hat{y}} \partial q_{y}} \\
& +\sum_{y} b_{y} \sum_{\hat{y}>y} a_{\hat{y}} \frac{\partial^{2} c_{\hat{y}}(\cdot)}{\partial q_{\hat{y}} \partial e_{y}}+2 \sum_{y} b_{y} \sum_{\hat{y}<y} b_{\hat{y}} \sum_{k>y} \frac{\partial^{2} c_{k}(\cdot)}{\partial e_{y} \partial e_{\hat{y}}}+\sum_{y} b_{y}^{2} \sum_{k>y} \frac{\partial^{2} c_{k}(\cdot)}{\partial e_{y}^{2}}
\end{aligned}
$$

The reduced index set of $b_{y}$ is implicitly covered in the summations. Plugging in the partial derivatives stated above and rearranging terms yields the following:

$$
\begin{aligned}
& \sum_{y} a_{y}^{2}\left(2 \beta_{y}+\gamma_{y} \frac{1}{\bar{q}+e(y)-q_{y}}\right)-2 \sum_{y} a_{y} \sum_{\hat{y}<y} b_{\hat{y}}\left(\gamma_{y} \frac{q_{y}}{\left(\bar{q}+e(y)-q_{y}\right)(\bar{q}+e(y))}\right) \\
& +2 \sum_{y} b_{y} \sum_{\hat{y}<y} b_{\hat{y}} \sum_{\tilde{y}>y}\left(\gamma_{\tilde{y}} \frac{q_{\tilde{y}}^{2}}{\left(\bar{q}+e(\tilde{y})-q_{\tilde{y}}\right)(\bar{q}+e(\tilde{y}))^{2}}\right) \\
& +\sum_{y} b_{y}^{2} \sum_{\tilde{y}>y}\left(\gamma_{\tilde{y}} \frac{q_{\tilde{y}}^{2}}{\left(\bar{q}+e(\tilde{y})-q_{\tilde{y}}\right)(\bar{q}+e(\tilde{y}))^{2}}\right)= \\
& =2 \sum_{y} a_{y}^{2} \beta_{y} \\
& +\sum_{y} \frac{\gamma_{y}}{\bar{q}+e(y)-q_{y}} \underbrace{\left(a_{y}^{2}-2\left(a_{y} \sum_{\hat{y}<y} b_{\hat{y}}\right) \frac{q_{y}}{\bar{q}+e(y)}+\sum_{\hat{y}<y}\left(2 b_{\hat{y}} \sum_{\tilde{y}<\hat{y}} b_{\tilde{y}}+b_{\hat{y}}^{2}\right) \frac{q_{y}^{2}}{(\bar{q}+e(y))^{2}}\right)}_{\circledast(y)}
\end{aligned}
$$


For positive semidefiniteness of the Hessian and hence convexity of the optimization problem, we require that the above term is non-negative for every feasible vector $(q, e)$. For the first part, $\sum_{y} a_{y}^{2} \beta_{y}$, that is straightforward for any vector $(a)$. The term $\frac{\gamma_{y}}{\bar{q}+e(y)-q_{y}}$ is non-negative in every period $y$, as otherwise the implicit production capacity constraint would be violated. For the last part, note that this term can actually be written as

$$
\circledast(y)=\left(a_{y}-\left(\sum_{\hat{y}<y} b_{\hat{y}}\right) \frac{q_{y}}{\bar{q}+e(y)}\right)^{2},
$$

hence it is always non-negative. Equation $(10)$ is thus non-negative for any positive cost parameter vectors $\alpha, \beta, \gamma, \kappa$ and any vector $x \in \mathbb{R}^{2 \bar{y}-1}$.

The proof allows one additional interesting observation: the upper left part of the Hessian matrix is positive definite for strictly positive cost parameters $\beta_{y}$, indicating that the quantity produced in equilibrium is unique in these cases. However, depending on the parameters, the part of the matrix pertaining to the investment decision may be either positive definite or positive semidefinite, depending on the parameters. This can be interpreted that there may be cases when the timing of the investment does not matter.

The following three corollaries show that the supplier's optimization problem with endogenous expansion decision variables can be easily included in the framework of the models discussed in the introduction: the assumption of price-taking behaviour (i.e., exogenous price $p_{y}$ ) may be relaxed and replaced by Cournot market power; engineering and other constraints may be considered in the optimization problem; and several suppliers may compete non-cooperatively, possibly in a richer equilibrium model with other types of players and price-sensitive demand.

Corollary 2. The exogenous price parameter $p_{y}$ may be replaced by an inverse demand function $P_{y}(\cdot)$, if it is linear and negatively sloped. Then Theorem 11 remains valid.

Proof. The Hessian matrix of this extended problem $\tilde{f}(q, e)$ is as follows:

$$
H(\tilde{f}(q, e))=H(f(q, e))+\operatorname{diag}\left(\left(-2 P_{y}^{\prime}(\cdot)\right)_{y \in\{1, \ldots, \bar{y}\}},(0)_{y \in\{1, \ldots, \bar{y}-1\}}\right)
$$

Since $P_{y}^{\prime}(\cdot)<0$, the second part of the extended Hessian is positive semidefinite. The sum of two positive semidefinite matrices is again positive semidefinite.

Corollary 3. Constraints of the form $g(q, e) \leq 0, h(q, e)=0$ may be added to the supplier's profit maximization problem (1) if $g(q, e)$ is convex and $h(q, e)$ is affine. Then Theorem 1 remains valid. 
Proof. The KKT conditions of an optimization problem of the form

$$
\min _{x} f(x) \text { s.t. } g(x) \leq 0, h(x)=0,
$$

with $f(x), g(x)$ convex and $h(x)$ affine are both necessary and sufficient.

Corollary 4. Equilibria between several suppliers with endogenous investment decisions and other players can be solved simultaneously as a MCP by taking the respective KKT conditions of each player, combined with appropriate market-clearing conditions, given that the other players' optimization problems are of a form such that their KKT conditions are sufficient for optimality.

Proof. Cf. Facchinei and Pang (2003).

\section{Conclusions and outlook}

This work provides a brief overview of current large-scale natural gas equilibrium models and points out their omission of endogenous investment decisions in production capacity, instead relying on exogenously determined capacity increases for future periods. I propose a mathematical formulation to incorporate capacity investments into the state-of-the-literature equilibrium models with logarithmic cost functions and show that this formulation is indeed a convex problem.

Nevertheless, implementing this formulation in a large-scale equilibrium model will increase the number of non-linear terms in the KKT conditions. While any solution obtained will indeed be an equilibrium, the logarithmic terms may lead to numerical problems and increased computation time. Therefore, the proposed formulation shall be integrated into a large-scale equilibrium model to test the numerical properties of this approach. In the future, the endogenous consideration of production capacity expansions may significantly improve the scope and validity of scenario simulations of the natural gas as well as other energy and resource markets.

\section{References}

Ibrahim Abada, Steven A. Gabriel, Vincent Briat, and Olivier Massol. A Generalized Nash-Cournot Model for the Northwestern European Natural Gas Markets with a Fuel Substitution Demand Function: The GaMMES Model. Networks and Spatial Economics, forthcoming, 2012.

EC. Energy Roadmap 2050. COM/2011/885. European Commission, 2011.

Ruud Egging, Franziska Holz, and Steven A. Gabriel. The World Gas Model - A Multi-Period Mixed Complementary Model for the Global Natural Gas Market. Energy, 35(10):4016-4029, 2010. 
Francisco Facchinei and Jong-Shi Pang. Finite-Dimensional Variational Inequalities and Complementarity Problems, volume I \& II. Springer, New York, 2003.

Michael C. Ferris and Todd S. Munson. Complementarity Problems in GAMS and the PATH Solver. Journal of Economic Dynamics and Control, 24(2):165-88, 2000.

Steven A. Gabriel, Jifang Zhuang, and Ruud Egging. Solving stochastic complementarity problems in energy market modeling using scenario reduction. European Journal of Operational Research, 197(3):1028-1040, 2009 .

Rolf Golombek, Eystein Gjelsvik, and Knut Einar Rosendahl. Effects of Liberalizing the Natural Gas Markets in Western Europe. Energy Journal, 16(1):85-112, 1995.

Patrick T. Harker and Jong-Shi Pang. Finite-dimensional variational inequality and nonlinear complementarity problems: A survey of theory, algorithms and applications. Mathematical Programming, 48(1):161-220, 1990.

Harald Hecking and Timo Panke. COLUMBUS - A global gas market model. EWI Working Paper 12/06, 2012.

IEA. World Energy Outlook 2011. Organisation for Economic Co-operation and Development, Paris, 2011.

Francois Leveque, Jean-Michel Glachant, Julian Barguin, Christian von Hirschhausen, Franziska Holz, and William J. Nuttall. Security of Energy Supply in Europe: Natural Gas, Nuclear and Hydrogen. E. Elgar Publishing, 2010.

Wietze Lise and Benjamin F. Hobbs. Future evolution of the liberalised European gas market: Simulation results with a dynamic model. Energy, 33(7):989-1004, 2008.

Lars Mathiesen. Computational Experience in Solving Equilibrium Models by a Sequence of Linear Complementarity Problems. Operations Research, 33(6):1225-1250, 1985.

Lars Mathiesen, Kjell Roland, and Knut Thonstad. The European Natural Gas Market: Degrees of Market Power on the Selling Side. In Rolf Golombek, Michael Hoel, and Jon Vislie, editors, Natural Gas Markets and Contracts. North-Holland, 1987.

Sauleh Siddiqui and Steven A. Gabriel. An SOS1-Based Approach for Solving MPECs with a Natural Gas Market Application. Networks and Spatial Economics, forthcoming, 2012. 